\title{
Toward the Optimum Measurement of Visual Acuity
}

\section{Tony Redmond}

School of Optometry and Vision Sciences, Cardiff University, Maindy Road, Cardiff, United Kingdom; RedmondT1@cardiff.ac.uk

The drawbacks of conventional letters in visual acuity measurements have been long acknowledged, including the nonuniform discriminability across the letter set ${ }^{1}$ and high test-retest variability. ${ }^{2}$ Apart from the introduction of the ETDRS chart, ${ }^{3}$ surprisingly few alterations have been made to the standard visual acuity test in the last 60 years. Although the Landolt $C$ target, with equal discriminability between different orientations, has been recognized as the gold standard for measuring visual acuity, ${ }^{4}$ this stimulus is not ideal for use in the clinical setting, owing in part to the small number of possible alternatives. Furthermore, "reading the letters on the chart" is arguably the most familiar test of vision to those undergoing an eye examination. With the advent of novel therapies for conditions such as age-related macular degeneration (AMD), what is increasingly needed is a letter chart with lower test-retest variability that does not require a change in task for the patient. Shah et al. ${ }^{5}$ propose the clinical use of vanishing optotypes, ${ }^{6}$ which may well meet these conditions and possibly provide more accurate measures of underlying functional retinal ganglion cell density than conventional letter stimuli, at least when viewed extrafoveally. These letter targets are pseudo-high-pass, in that they have dark strokes with light edges or vice versa and a mean luminance equal to the surround. Detection and recognition are equal in the fovea; the targets vanish as soon as the recognition limit is reached.

The authors measured detection and recognition acuity for conventional and vanishing optotypes in the fovea and at $10^{\circ}$ eccentricity in two psychophysically trained healthy individuals, with and without optical blur. The article reports several key findings; while comparable in the fovea, a separation between detection and recognition acuity for "vanishing" optotypes became apparent outside the fovea. This, together with a relative robustness of peripheral recognition acuity to optical blur, lends support to the notion that recognition acuity for these targets is sampling limited outside the fovea. Furthermore, and perhaps most important clinically, the authors confirm previous findings ${ }^{7}$ that discriminability is more uniform across the entire letter set when low spatial frequency content is removed. They conclude that more repeatable visual acuity measurements may be obtained with vanishing optotypes than with conventional letters.

Further experiments are required to explore the performance of vanishing optotypes in naive observers in research and clinical settings, in clinical trials, and in disease, but these initial findings are encouraging for an improvement on current methods without altering the task of the patient.

\section{References}

1. Reich LN, Bedell HE. Relative legibility and confusions of letter acuity targets in the peripheral and central retina. Optom Vis Sci. 2000; 77:270-275.

2. Arditi A, Cagenello R. On the statistical reliability of letter-chart visual acuity measurements. Invest Ophthalmol Vis Sci. 1993;34:120129

3. Ferris FL III, Kassoff A, Bresnick GH, Bailey I. New visual acuity charts for clinical research. Am J Ophthalmol. 1982;94:91-96.

4. National Research Council Committee on Vision. Recommended standard procedures for the clinical measurement and specification of visual acuity. Adv Ophthalmol. 1980;41:103-148.

5. Shah N, Dakin SC, Anderson RS. Effect of optical defocus on detection and recognition of vanishing optotype letters in the fovea and periphery. Invest Ophthalmol Vis Sci. 2012;53:7063-7070.

6. Howland B, Ginsburg A, Campbell F. High-pass spatial frequency letters as clinical optotypes. Vision Res. 1978;18:1063-1066.

7. Shah N, Dakin SC, Redmond T, Anderson RS. Vanishing optotype acuity: repeatability and effect of the number of alternatives Ophthalmic Physiol Opt. 2011;31:17-22. 